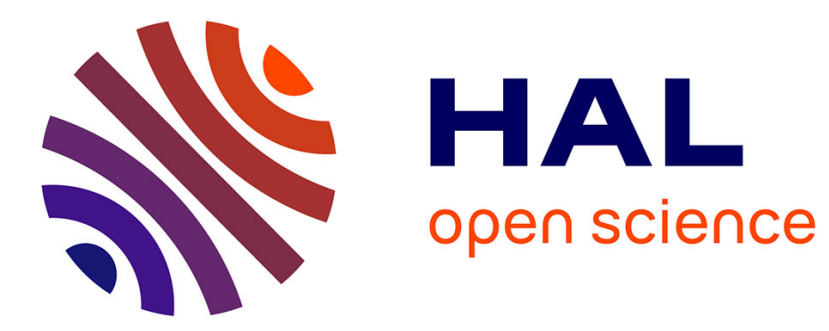

\title{
A simple approach to the state-specific MR-CC using the intermediate Hamiltonian formalism
}

Emmanuel Giner, Grégoire David, Anthony Scemama, Jean-Paul Malrieu

\section{To cite this version:}

Emmanuel Giner, Grégoire David, Anthony Scemama, Jean-Paul Malrieu. A simple approach to the state-specific MR-CC using the intermediate Hamiltonian formalism. Journal of Chemical Physics, 2016, 144 (6), pp.064101. 10.1063/1.4940781 . hal-01298011

\section{HAL Id: hal-01298011 \\ https://hal.science/hal-01298011}

Submitted on 29 Jan 2020

HAL is a multi-disciplinary open access archive for the deposit and dissemination of scientific research documents, whether they are published or not. The documents may come from teaching and research institutions in France or abroad, or from public or private research centers.
L'archive ouverte pluridisciplinaire HAL, est destinée au dépôt et à la diffusion de documents scientifiques de niveau recherche, publiés ou non, émanant des établissements d'enseignement et de recherche français ou étrangers, des laboratoires publics ou privés. 


\section{A simple approach to the state-specific MR- CC using the intermediate Hamiltonian formalism}

Cite as: J. Chem. Phys. 144, 064101 (2016); https://doi.org/10.1063/1.4940781

Submitted: 10 September 2015 . Accepted: 14 January 2016 . Published Online: 09 February 2016

E. Giner (D), G. David, A. Scemama (D), and J. P. Malrieu
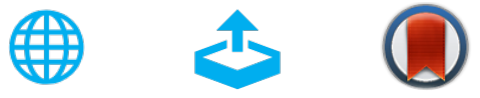

\section{ARTICLES YOU MAY BE INTERESTED IN}

Alternative definition of excitation amplitudes in multi-reference state-specific coupled cluster

The Journal of Chemical Physics 146, 154107 (2017); https://doi.org/10.1063/1.4980034

A Jeziorski-Monkhorst fully uncontracted multi-reference perturbative treatment. I. Principles, second-order versions, and tests on ground state potential energy curves The Journal of Chemical Physics 146, 224108 (2017); https://doi.org/10.1063/1.4984616

Stochastic multi-reference perturbation theory with application to the linearized coupled cluster method

The Journal of Chemical Physics 146, 044107 (2017); https://doi.org/10.1063/1.4974177

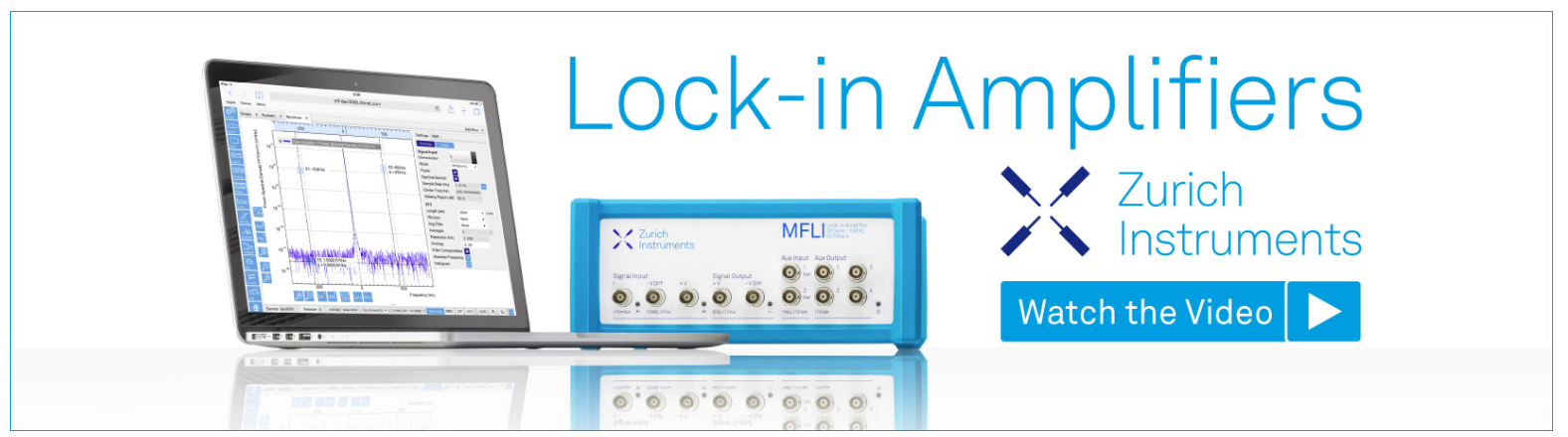




\title{
A simple approach to the state-specific MR-CC using the intermediate Hamiltonian formalism
}

\author{
E. Giner, ${ }^{1}$ G. David, ${ }^{2}$ A. Scemama, ${ }^{2, a)}$ and J. P. Malrieu ${ }^{2}$ \\ ${ }^{1}$ Dipartimento di Scienze Chimiche e Farmaceutiche, Universita di Ferrara, Via Fossato di Mortara 17, \\ I-44121 Ferrara, Italy \\ ${ }^{2}$ Laboratoire de Chimie et Physique Quantiques (CNRS 5626), IRSAMC, Université P. Sabatier, \\ Toulouse, France
}

(Received 10 September 2015; accepted 14 January 2016; published online 9 February 2016)

This paper presents a rigorous state-specific multi-reference coupled cluster formulation of the method first proposed by Meller et al. [J. Chem. Phys. 104, 4068 (1996)]. Guess values of the amplitudes of the single and double excitations (the $\hat{\mathcal{T}}$ operator) on the top of the references are extracted from the knowledge of the coefficients of the Multi-Reference Singles and Doubles Configuration Interaction (MR-CISD) matrix. The multiple parentage problem is solved by scaling these amplitudes from the interaction between the references and the singles and doubles. Then one proceeds to a dressing of the MR-CISD matrix under the effect of the triples and quadruples, the coefficients of which are estimated from the action of $\exp (\hat{\mathcal{T}})$. This dressing follows the logic of the intermediate effective Hamiltonian formalism. The dressed MR-CISD matrix is diagonalized and the process is iterated to convergence. As a simplification, the coefficients of the triples and quadruples may in practice be calculated from the action of $\hat{\mathcal{T}}^{2}$ only, introducing 5th-order differences in the energies. The so-simplified method is tested on a series of benchmark systems from Complete Active Spaces (CASs) involving 2-6 active electrons up to bond breakings. The comparison with full configuration interaction results shows that the errors are of the order of a few millihartree, five times smaller than those of the CAS-CISD, and the deviation to strict separability is lower than $10 \mu$ hartree. The method is totally uncontracted, parallelizable, and extremely flexible since it may be applied to selected MR and/or selected CISD. Some potential generalizations are briefly discussed. ( 2016 AIP Publishing LLC. [http://dx.doi.org/10.1063/1.4940781]

\section{INTRODUCTION}

In the domain of molecular physics and quantum chemistry, the many-body problem is perfectly clear as long as it is formulated from a single reference. The perturbative expansion of the wave operator and its diagrammatic transcription offers a guide to understanding the relation between the multiplicative structure of the wave function and the additive structure of the energy. The linked cluster theorem ${ }^{1}$ clarifies the questions of the size consistency and of the strict separability into fragments. The defects of truncated Configuration Interaction (CI) are well understood and algorithms have been proposed to respect approximately (CEPA- $\left.0,{ }^{2,3} \mathrm{CEPA}-n^{4-6}\right)$ or strictly $\left((\mathrm{SC})^{2} \mathrm{CI}\right)^{7}$ the cancellation of unlinked diagrams. The Coupled Cluster (CC) method ${ }^{8-10}$ is definitely the most elegant formalism and can be considered as the standard treatment in its Coupled Cluster Singles and Doubles (CCSD) version, or in the CCSD(T) version, ${ }^{11}$ which incorporates the fourth-order effect of the triply excited determinants. But all these approaches fail when one cannot expect that a single determinant will represent a reliable starting point to conveniently generate the wave function.

This is precisely the situation in many domains. The excited states present an intrinsic multi-determinantal character, and frequently a multi-configurational character.

\footnotetext{
a)Electronic mail: scemama@irsamc.ups-tlse.fr
}

Such are the magnetic systems in their low energy states, and the treatment of chemical reactions, in which chemical bonds are broken, also requires to consider geometries for which a single determinant picture is not relevant. A generalized linked cluster theorem has been established by Brandow, ${ }^{12}$ which gives a conceptual guide, but the conditions that must be fulfilled for its demonstration (Complete Active Space (CAS) as reference space, mono-electronic zero-order Hamiltonian) would lead to strongly divergent behaviors of the corresponding perturbative expansion in any realistic molecular problem. Practical computational tools have been proposed, most of them being state-specific. One may quote second order perturbation expansions based on determinants from selected references (Configuration Interaction Perturbing a multi-configurational zeroth-order wave function Selected Iteratively, CIPSI), ${ }^{13,14}$ intermediate Hamiltonian dressing, ${ }^{15}$ in the so-called shifted $B_{k}$ technique. ${ }^{16}$ These methods are not strictly size-consistent, and the conditions to satisfy the strict separability of determinant-based expansions require to define sophisticated zero-order Hamiltonians. ${ }^{17}$ Contracted perturbative expansions, which perturb the multideterminant zero-order wave function under the effect of linear combinations of outer-space determinants, have also been proposed. One may quote the CASPT2 method $^{18,19}$ which uses a mono-electronic zero-order Hamiltonian, faces intruder state problems, and is not size consistent; the NEVPT2 method ${ }^{20-22}$ which uses a bi-electronic zero-order Hamiltonian 
(the Dyall's one ${ }^{23}$ ) and is size consistent and intruder-state free; the method from Werner; ${ }^{24}$ as well as the perturbation derived by Mukherjee et $a .^{25-27}$ from their multi-reference coupled cluster (MR-CC) formalism.

If the CASSCF wave function is considered as the counterpart of the single-determinant reference, the CASCISD is the counterpart of the CISD, with the same sizeinconsistence defect, and the research of MR-CEPA and MR$\mathrm{CC}$ has been the subject of intense methodological researches for about 20 years, without evident success. The cancellation of all unlinked terms in the MR expansion (i.e., a MR-CEPA or MR-(SC) $\left.)^{2}-\mathrm{CI}\right)$ formalism is not an easy task. ${ }^{28,29}$ If one lets aside the MR-CC methods that attribute a specific role to a single reference, ${ }^{30}$ a few state-specific strictly multi-reference $\mathrm{CC}$ methods have been proposed: one suggested by one of the authors and collaborators, ${ }^{31}$ another one by Mukherjee and co-workers ${ }^{32}$ which has been intensively tested, and a third one in a Brillouin Wigner context. ${ }^{33,34}$ We return here on the first proposal which was only tested on a single problem. We shall present an improved formulation of the method in Section II, then the principle of its implementation (Section III), followed by numerical illustrations of its accuracy (Section IV). Section V comments on the properties of the method and Section VI discusses the advantages of this formalism, its flexibility, and possible extensions.

\section{METHOD}

\section{A. Principle}

Let us call $|I\rangle$ the reference determinants, the number of which will be called $N$. The reference space may be a CAS, but this is only compulsory if one wants to satisfy the strict-separability property. If not the method is applicable to incomplete model spaces as well. The projector on the model space is

$$
\hat{P}_{0}=\sum_{I}|I\rangle\langle I|
$$

Let us consider a zero-order wave function restricted to the model space,

$$
\left|\Psi_{0}^{m}\right\rangle=\sum_{I} c_{I}^{m}|I\rangle
$$

This function may be either the eigenfunction of $\hat{P}_{0} \hat{H} \hat{P}_{0}$,

$$
\hat{P}_{0} \hat{H} \hat{P}_{0}\left|\Psi_{0}^{m}\right\rangle=E_{0}^{m}\left|\Psi_{0}^{m}\right\rangle,
$$

or the projection of the eigenvector of the CAS-CISD on the model space,

$$
\left|\Psi_{0}^{m}\right\rangle=\hat{P}_{0}\left|\Psi_{\mathrm{CAS}-\mathrm{CISD}}^{m}\right\rangle .
$$

The CAS-CISD wave function is written as

$$
\left|\Psi_{\mathrm{CAS}-\mathrm{CISD}}^{m}\right\rangle=\sum_{I} c_{I}^{m}|I\rangle+\sum_{i} c_{i}^{m}|i\rangle,
$$

where $|i\rangle$ are the singles and doubles (the determinants of the CAS-CISD space which do not belong to the reference space). We want to follow the Jeziorski-Monkhorst ${ }^{35}$ expression of $^{3}$ the wave operator $\hat{\Omega}^{m}$ which is supposed to transform the zero-order wave function into the exact one,

$$
\hat{\Omega}^{m}\left|\Psi_{0}^{m}\right\rangle=\left|\Psi^{m}\right\rangle
$$

as a sum of reference-dependent operators,

$$
\hat{\Omega}^{m} \hat{P}_{0}=\sum_{I} \hat{\Omega}_{I}^{m}|I\rangle\langle I| .
$$

Each of the $\hat{\Omega}_{I}^{m}$ 's will take an exponential form

$$
\hat{\Omega}_{I}^{m}=\exp \left(\hat{\mathcal{T}}_{I}^{m}\right)
$$

and each operator $\hat{\mathcal{T}}_{I}^{m}$ will be truncated to the single and double excitations, as one does in the CCSD formalism.

\section{B. The multi-parentage problem and the extraction of guess values of the excitation amplitudes from the CAS-CISD eigenvector}

One may easily recognize that there exist some degrees of freedom in the determination of the wave operators. In the single reference CCSD expansion, one searches for the amplitudes of the excitations sending from the reference $\Phi_{0}$ to the singly and doubly excited determinants. One evaluates the amplitudes of the triples and quadruples as given by the action of $\hat{\mathcal{T}}^{2}$ on $\Phi_{0}$, and the eigenequation is projected on each of the singles and doubles. If the number of singles and doubles is $n$, one may write a set of $n$ coupled quadratic equations on the amplitudes. But it may be convenient to guess a first evaluation of these amplitudes from the coefficients of the singles and doubles in the CISD matrix, which may be done in a unique manner. From these amplitudes one may obtain a guess of the coefficients of the triples and quadruples and it is convenient (ensuring for instance a better convergence than solving coupled biquadratic equation) to write the process as an iterative dressing of the CISD matrix, in the spirit of Intermediate Effective Hamiltonian formalism. ${ }^{15}$ This is a procedure to solve the set of non-linear equations and the results are identical to those obtained with the traditional formulation of the CCSD method.

In the multi-reference context one faces a genealogical problem, sometimes called the multiple-parentage problem. Actually for a state-specific formalism, one has only one coefficient for each of the singly and doubly excited determinants $|i\rangle$. In principle one may decide that this determinant is obtained from each of the references and one would then write

$$
c_{i}^{m}=\sum_{I} \tilde{d}_{I i}^{m} c_{I}^{m}
$$

but one must find a criterion to define the $N \tilde{d}_{I i}$ amplitudes from the knowledge of a single coefficient. Returning to a perturbative estimate of the coefficients of the singles and doubles starting from $\Psi_{0}^{m}$, the first-order expression of these coefficients,

$$
c_{i}^{m(1)}=\frac{\left\langle\Psi_{0}^{m}|\hat{H}| i\right\rangle}{E_{0}^{m}-\langle i|\hat{H}| i\rangle}=\sum_{I} c_{I}^{m} \frac{\langle I|\hat{H}| i\rangle}{E_{0}^{m}-\langle i|\hat{H}| i\rangle},
$$


suggests that the amplitudes of the excitation operators from the references to the singles and doubles might satisfy

$$
\frac{\tilde{d}_{I i}^{m}}{\tilde{d}_{J i}^{m}}=\frac{\langle I|\hat{H}| i\rangle}{\langle J|\hat{H}| i\rangle} .
$$

This scaling had been proposed in Ref. 31. This condition may be expressed as

$$
\tilde{d}_{I i}^{m}=\lambda_{i}^{m}\langle I|\hat{H}| i\rangle,
$$

where the quantity $\lambda_{i}^{m}$ is the inverse of an energy. Re-injecting this expression in Eq. (9) leads to

$$
c_{i}^{m}=\lambda_{i}^{m} \sum_{I} c_{I}^{m}\langle I|\hat{H}| i\rangle,
$$

which defines $\lambda_{i}^{m}$ as

$$
\lambda_{i}^{m}=\frac{c_{i}^{m}}{\left\langle\Psi_{0}^{m}|\hat{H}| i\right\rangle} .
$$

These are the key equations which define guess values of the amplitudes of the excitations leading from the references to the singles and doubles. Notice that we only consider amplitudes for the excitations which correspond to physical interactions, and since $\hat{H}$ is at most bi-electronic, one only introduces single- and double-excitation operators. Finally, we can re-express the CAS-CISD wave function as

$$
\left|\Psi_{\mathrm{CAS}-\mathrm{CISD}}^{m}\right\rangle=\sum_{I} c_{I}^{m}\left(1+\sum_{i} \tilde{d}_{I i}^{m} \hat{T}_{I i}\right)|I\rangle,
$$

where

$$
\hat{T}_{I i}|I\rangle=|i\rangle
$$

In order to reach the coupled cluster formalism, it is necessary to subtract the contribution of the product of single amplitudes from the amplitudes $\tilde{d}_{I i}^{m}$,

$$
\begin{aligned}
c_{i}^{m}= & \sum_{I} \tilde{d}_{I i}^{m} c_{I}^{m}=\sum_{I \in \operatorname{Singles}(i)} s_{I i}^{m} c_{I}^{m} \\
& +\sum_{I \in \operatorname{Doubles}(i)}\left(d_{I i}^{m}+\sum_{(k, l) \in I \rightarrow i} \pm s_{I k}^{m} s_{I l}^{m}\right) c_{I}^{m},
\end{aligned}
$$

where

- $s_{I i}^{m}$ are the amplitudes of the single excitation operators between the reference $|I\rangle$ and $|i\rangle$,

- $d_{I i}^{m}$ are the amplitudes of the double excitation operators between the reference $|I\rangle$ and $|i\rangle$,

- $(k, l) \in(I \rightarrow i)$ denotes the couples $(k, l)$ for which $\hat{T}_{I k} \hat{T}_{I l}= \pm \hat{T}_{I i}$, the sign being governed by the permutation logics.

The single excitation amplitudes $s_{I i}^{m}$ are simply given by $s_{I i}^{m}=\tilde{d}_{I i}^{m}$, and the double excitation amplitudes are obtained from Eq. (17),

$$
d_{I i}^{m}=\tilde{d}_{I i}^{m}-\sum_{(k, l) \in I \rightarrow i} \pm s_{I k}^{m} s_{I l}^{m} .
$$

\section{Coefficients of the triples and quadruples}

One may then generate the triples and quadruples $|\alpha\rangle$. Among them only those which interact with the singles and doubles (i.e., which are generated by the action of $\hat{H}$ on the singles and doubles and which do not belong to the CAS-CISD space) have to be considered. One may find the references with which they present either 3 or 4 differences in the occupation numbers of the molecular orbitals (MOs). These reference determinants may be called the grand-parents of $|\alpha\rangle$. The comparison between $|\alpha\rangle$ and each of its grand-parents $|I\rangle$ defines the excitation operator from $|I\rangle$ to $|\alpha\rangle$ as a triple or quadruple excitation,

$$
\hat{T}_{I \alpha}|I\rangle=|\alpha\rangle,
$$

which may be expressed in second quantization as the product of 4 (or 3) creation operators and 4 (or 3) annihilation operators,

$$
\hat{T}_{I \alpha}=a_{q}^{\dagger} a_{p}^{\dagger} a_{n}^{\dagger} a_{l}^{\dagger} a_{e} a_{f} a_{g} a_{h} .
$$

The creations run on active and virtual MOs, the annihilations run on active and inactive occupied MOs but the number of inactive indices among the creation and/or among the particles must be equal to 3 or 4 , otherwise the determinant would belong to the CAS-CISD space. Knowing the operator, it may be factorized as the product of all complementary double or single excitation operators in all possible manners (each excitation keeping untouched the $M_{s}$ value). Then we may write the contribution to the coefficient of $|\alpha\rangle$ differing by 3 orbitals from $|I\rangle$ as

$$
d_{I \alpha}^{m}=\sum_{(k, l) \in(I \rightarrow \alpha)} \pm d_{I k}^{m} s_{I l}^{m}+\sum_{(j, k, l) \in(I \rightarrow \alpha)} \pm s_{I j}^{m} s_{I k}^{m} s_{I l}^{m}
$$

and similarly if they differ by 4 orbitals,

$$
\begin{aligned}
d_{I \alpha}^{m}= & \sum_{(k, l) \in(I \rightarrow \alpha)} \pm d_{I k}^{m} d_{I l}^{m}+\sum_{(j, k, l) \in(I \rightarrow \alpha)} \pm d_{I j}^{m} s_{I k}^{m} s_{I l}^{m} \\
& +\sum_{(i, j, k, l) \in(I \rightarrow \alpha)} \pm s_{I i}^{m} s_{I J}^{m} s_{I k}^{m} s_{I l}^{m} .
\end{aligned}
$$

Finally, one may write the coefficient $c_{\alpha}^{m}$ as

$$
c_{\alpha}^{m}=\sum_{I} d_{I \alpha}^{m} c_{I}^{m} .
$$

\section{Dressing of the CAS-CISD matrix}

If one considers the eigenequation relative to $\langle i|$,

$$
\begin{aligned}
\left(\langle i|\hat{H}| i\rangle-E^{m}\right) c_{i}^{m} & +\sum_{I}\langle i|\hat{H}| I\rangle c_{I}^{m}+\sum_{j \neq i}\langle i|\hat{H}| j\rangle c_{j}^{m} \\
& +\sum_{\alpha}\langle i|\hat{H}| \alpha\rangle c_{\alpha}^{m}=0
\end{aligned}
$$

one may decompose the last term,

$$
\begin{aligned}
\sum_{\alpha}\langle i|\hat{H}| \alpha\rangle c_{\alpha}^{m} & =\sum_{\alpha}\langle i|\hat{H}| \alpha\rangle \sum_{I} d_{I \alpha}^{m} c_{I}^{m} \\
& =\sum_{I}\left(\sum_{\alpha} d_{I \alpha}^{m}\langle i|\hat{H}| \alpha\rangle\right) c_{I}^{m} .
\end{aligned}
$$


Introducing the quantities,

$$
\left\langle i\left|\hat{\Delta}^{m}\right| I\right\rangle=\sum_{\alpha} d_{I \alpha}^{m}\langle i|\hat{H}| \alpha\rangle,
$$

one may write the eigenequation (24) as

$$
\begin{aligned}
\left(\langle i|\hat{H}| i\rangle-E^{m}\right) c_{i}^{m} & +\sum_{I}\left(\langle i|\hat{H}| I\rangle+\left\langle i\left|\hat{\Delta}^{m}\right| I\right\rangle\right) c_{I}^{m} \\
& +\sum_{j \neq i}\langle i|\hat{H}| j\rangle c_{j}^{m}=0
\end{aligned}
$$

which suggests to treat the effect of the triples and quadruples as a column dressing of the CAS-CISD matrix. A similar idea has been exploited in the single-reference CCSD context, which may be presented and managed as an iterative dressing of the column between the reference and the singles and doubles. ${ }^{36}$ The coupled cluster dressed CAS-CISD Hamiltonian may be written as $\hat{P}_{\text {CAS-CISD }}\left(\hat{H}+\hat{\Delta}^{m}\right) \hat{P}_{\text {CAS-CISD, }}$ which is non-Hermitian. Defining the projector on the singles and doubles as

$$
\begin{gathered}
\hat{P}_{\mathrm{SD}}=\hat{P}_{\mathrm{CAS}-\mathrm{CISD}}-\hat{P}_{0}, \\
\hat{P}_{\mathrm{CAS}-\mathrm{CISD}} \hat{\Delta}^{m} \hat{P}_{\mathrm{CAS-CISD}}=\hat{P}_{\mathrm{SD}} \hat{\Delta}^{m} \hat{P}_{0},
\end{gathered}
$$

one may define an equivalent Hermitian dressing $\hat{\Delta}^{m \prime}$ in the case where one considers the Hermitization of the dressed CAS-CISD matrix to be desirable,

$$
\left\langle i\left|\hat{\Delta}^{m \prime}\right| I\right\rangle=\left\langle I\left|\hat{\Delta}^{m \prime}\right| i\right\rangle=\left\langle i\left|\hat{\Delta}^{m}\right| I\right\rangle
$$

provided that one introduces a diagonal dressing of the CASCISD matrix,

$$
\left\langle I\left|\hat{\Delta}^{m \prime}\right| I\right\rangle=-\frac{1}{c_{I}^{m}}\left(\sum_{i}\left\langle I\left|\hat{\Delta}^{m \prime}\right| i\right\rangle c_{i}^{m}\right) .
$$

The diagonalization of the matrices

$$
\hat{P}_{\text {CAS-CISD }}\left(\hat{H}+\hat{\Delta}^{m}\right) \hat{P}_{\text {CAS-CISD }}
$$

and

$$
\hat{P}_{\text {CAS-CISD }}\left(\hat{H}+\hat{\Delta}^{m \prime}\right) \hat{P}_{\text {CAS-CISD }}
$$

will give the same desired eigenenergy and eigenvector

$$
\begin{aligned}
& \hat{P}_{\mathrm{CAS}-\mathrm{CISD}}\left(\hat{H}+\hat{\Delta}^{m}\right) \hat{P}_{\mathrm{CAS}-\mathrm{CISD}}\left|\Psi_{\mathrm{CC}}^{m}\right\rangle \\
& \quad=E_{\mathrm{CC}}^{m} \hat{P}_{\mathrm{CAS}-\mathrm{CISD}}\left|\Psi_{\mathrm{CC}}^{m}\right\rangle, \\
& \hat{P}_{\mathrm{CAS}-\mathrm{CISD}}\left(\hat{H}+\hat{\Delta}^{m \prime}\right) \hat{P}_{\mathrm{CAS}-\mathrm{CISD}}\left|\Psi_{\mathrm{CC}}^{m}\right\rangle \\
& \quad=E_{\mathrm{CC}}^{m} \hat{P}_{\mathrm{CAS}-\mathrm{CISD}}\left|\Psi_{\mathrm{CC}}^{m}\right\rangle .
\end{aligned}
$$

Of course the process has to be iterated, and the resulting eigenvector defines new coefficients on both the references and the singles and doubles, which lead to new amplitudes, new evaluations of the coefficients of the triples and quadruples, and new dressings. Since the eigenvectors of the dressed matrices are identical, the two formulations, Hermitian or non-Hermitian, converge to the same solution. The converged solutions are the MR-CCSD energy and the MR-CCSD amplitudes, which define the exponential wave operator. One should keep in mind that the building of the dressed CAS-CISD matrix is not the only way to solve the non-linear equations of the MR$\mathrm{CC}$ formalism, and more standard techniques like the Newton method or DIIS might be applied here.

\section{E. A convenient approximation}

The coupled cluster expansion can be related to a perturbation expansion, for instance, in terms of diagrammatic development of the wave function. Considering only the second power of $\hat{\mathcal{T}}$ in the evaluation of the coefficients of the triples and quadruples ensures that these coefficients are correct to the second-order and that the energy is correct to the fourth-order. Hence, the second sum of (21) and the two last sums of (22) introduce only contributions of the third and fourth orders to the wave function, and of the fifth and sixth orders to the energy. Consistently, if these terms are neglected, one obtains $d_{I i}^{m}=\tilde{d}_{I i}^{m}$ and a simplified expression of $d_{I \alpha}^{m}$,

$$
d_{I \alpha}^{m}=\sum_{(k, l) \in(I \rightarrow \alpha)} \pm \tilde{d}_{I k}^{m} \tilde{d}_{I l}^{m}
$$

Splitting the excitation operators $\hat{\mathcal{T}}$ into single and double excitation operators, $\hat{\mathcal{T}}_{1}$ and $\hat{\mathcal{T}_{2}}$ respectively, the terms $\left(\hat{\mathcal{T}_{1}}\right)^{2}$, $\hat{\mathcal{T}_{1}} \hat{\mathcal{T}}_{2}$, and $\left(\hat{\mathcal{T}}_{2}\right)^{2}$ are correctly treated. The errors with respect to the full coupled cluster expansion arise from the $\left(\hat{\mathcal{T}}_{1}\right)^{2} \hat{\mathcal{T}}_{2}$, $\left(\hat{\mathcal{T}}_{1}\right)^{3}$, and $\left(\hat{\mathcal{T}}_{1}\right)^{4}$. The price to pay is a certain violation of the strict separability, i.e., of the additivity of the energies of two subsystems $A$ and $B$ when treating the $A B$ system at infinite distances. This will be illustrated in Section V B.

\section{IMPLEMENTATION}

The proposed algorithm was implemented in the Quantum Package ${ }^{37}$ an open-source series of programs developed in our laboratory. The bottleneck of this algorithm is the determinant comparisons needed to determine the excitation operators and phases during the reconstruction of the genealogy of the $|\alpha\rangle$ 's. This was made possible, thanks to a very efficient implementation of Slater-Condon's rules. ${ }^{38}$

\section{A. General structure}

At each iteration step, one first assigns the values of the $\lambda_{i}^{m}$ parameters obtained from the eigenvector of the (dressed) CAS-CISD matrix according to Equation (14). From these parameters $\lambda_{i}^{m}$, the amplitudes of the single and double excitations are uniquely defined. Then one loops on the singles and doubles $|i\rangle$. On each of them, one reapplies the excitation operators to generate the $|\alpha\rangle$ 's. Those which belong to the CAS-CISD space are eliminated. The parents of $|\alpha\rangle$ (that are all the singles and doubles $|k\rangle$ 's such that $\langle\alpha|\hat{H}| k\rangle \neq 0$ ) are generated. If one of the $|k\rangle$ 's has already been considered in the loop on the $|i\rangle$ 's $(k<i)$, this $|\alpha\rangle$ has been already generated and taken into account and must not be double counted. While generating the parents of $|\alpha\rangle$, its interactions with them, $\langle k|\hat{H}| \alpha\rangle$, are stored. At this step, the reference grand-parents $|I\rangle$ are identified as having 3 or 4 differences with $|\alpha\rangle$. Then, the excitation operator leading from $|I\rangle$ to $|\alpha\rangle$ is expressed in all possible manners as products of two complementary single or double excitations. For each couple of complementary excitations, the product of the amplitudes is accumulated to compute $d_{I \alpha}^{m}$ according to Equation (34). Finally, the product 
of $\langle k|\hat{H}| \alpha\rangle$ with $d_{I \alpha}^{m}$ is accumulated in $\left\langle k\left|\hat{\Delta}^{m}\right| I\right\rangle$ for each parent $|k\rangle$ of $|\alpha\rangle$ according to Equation (26). Once the loop on the $|i\rangle$ 's is done, all the $|\alpha\rangle$ 's have been generated, and the column dressing is completed. Then, in order to fit with a symmetric diagonalization technique, one symmetrizes the dressing as mentioned in Sec. II (Equations (30) and (31)). The dressed CAS-CISD matrix is diagonalized and the process is repeated up to convergence of the calculated dressed energy. From the computational point of view, this process requires the storing of the dressing columns which scales as $N \times\left(n_{\text {occ }} n_{\text {virt }}\right)^{2}$ where $N$ is the number of determinants in the reference, and $n_{\text {occ }}$ and $n_{\text {virt }}$ are, respectively, the number of occupied and virtual MOs. This amount of memory is reasonable and does not represent a bottleneck for the present applications. Regarding the CPU time, the costly part concerns the handling of the $|\alpha\rangle$ 's which scales as $\left(n_{\text {occ }} n_{\text {virt }}\right)^{4}$. Nevertheless, the process is perfectly parallelizable as all the work done with the $|\alpha\rangle$ 's generated from $|i\rangle$ does not depend on any other $|\alpha\rangle$.

\section{B. Practical issues}

The definition of $\lambda_{i}^{m}$ can lead to numerical instabilities when $\left\langle\Psi_{0}^{m}|\hat{H}| i\right\rangle$ is small. Nevertheless, in such cases the contribution of $|i\rangle$ to the post-CAS correlation energy is also small, suggesting that one might use a perturbative estimate of $\lambda_{i}^{m}$. In practice, we use the perturbative $\lambda_{i}$ according to two different criteria. The first one concerns the ratio of the variational coefficient $c_{i}^{m}$ (obtained at a given iteration) over its perturbative estimate (see (10)). If $\frac{c_{i}^{m(1)}}{c_{i}^{m}} \notin[0,0.5]$ then the amplitudes involving $|i\rangle$ are determined using the perturbative $\lambda_{i}^{m \text { (pert) }}$ defined as

$$
\lambda_{i}^{m(\mathrm{pert})}=\frac{1}{E_{0}^{m}-\langle i|\hat{H}| i\rangle} .
$$

In such situations, the coefficient $c_{i}^{m}$ is not determined by its interaction with the reference determinants, but comes from higher-order effects. The second criterion concerns the absolute value of each of the $d_{I i}^{m}$ defined according to (12). If any of these terms calculated with the $\lambda_{i}^{m}$ obtained from the variational calculation (see (14)) is larger than 0.5 , the perturbative $\lambda_{i}^{m \text { (pert) }}$ is used to determine the amplitudes $d_{I i}^{m \text { (pert) }}$ defined as

$$
d_{I i}^{m(\text { pert })}=H_{I i} \lambda_{i}^{m(\text { pert })}
$$

and the working amplitudes $d_{I i}^{m}$ are set to $d_{I i}^{m \text { (pert) }}$. This condition avoids numerical instabilities occurring when both $c_{i}^{m}$ and $\left\langle\psi_{0}^{m}|\hat{H}| i\right\rangle$ are small and allows us the control of the maximum value of the amplitudes. As soon as along the iterations one of the $|i\rangle$ 's fulfills one of these criteria, it will be treated perturbatively in the following iterations. This precaution avoids significant oscillations due to back and forth movements from perturbative to variational treatment of the $\lambda_{i}^{m}$. The numerically observed residual oscillations are of the order of magnitude of $10^{-6} E_{\mathrm{h}}$, which may certainly be attributed to the non linear character of the numerical algorithm. Nevertheless, the order of magnitude of the residual oscillations is much smaller than the chemical and even spectroscopic accuracy. Similar instability problems have been noticed in the Mk-MRCC treatment. ${ }^{39,40}$

\section{NUMERICAL TEST STUDIES}

We decided to test the accuracy and robustness of the method on a series of benchmarks, some of which have been used in the evaluations of other MR-CC proposals and of alternative MR approaches. They essentially concern model problems, especially bond breaking problems or the treatment of degenerate situations. They require to use a CAS with up to six electrons in six MOs. In all cases, the method converged in a few iterations. A systematic comparison is made with full configuration interaction (FCI) estimates, either taken from the literature or obtained from a CIPSI type variation+perturbation calculation ${ }^{13,14}$ where the perturbative residue is about $-6 \mathrm{~m} E_{\mathrm{h}}$. Of course, the CAS-CISD is already a rather sophisticated treatment, which takes into account, although in a size-inconsistent manner, the leading correlation effects, both the non-dynamical part in the CAS and the dynamical part in the CISD step. One may expect that the improvement brought by the MR-CC treatment will be significant when the number of important inactive double excitations is large.

In order to have a global view of the performance of the here-proposed algorithm, we report potential energy curves and the error to FCI estimate of our MR-CCSD algorithm together with the CAS-CISD. Tables showing the error with respect to the FCI estimate of the MR-CCSD and CAS-CISD are also presented, complemented by the total energies of the FCI estimate. The non-parallelism error (NPE) is here calculated as the difference between the minimum and maximum error to the FCI estimate. The spectroscopic constants are obtained from an accurate fit of the obtained potential energy curves with a generalized Morse potential representation. The spectroscopic constants reported here are the equilibrium distance $R_{\text {eq }}$ in $\AA$, the frequency $k_{\text {eq }}$ in $E_{\mathrm{h}} / \AA^{2}$, and the atomization energy $D_{e}$ in $\mathrm{kcal} / \mathrm{mol}$.

CAS-SCF orbitals were obtained with the GAMESS(US) program $^{41}$ and all Multi-Reference Singles and Doubles Configuration Interaction (MR-CISD), CIPSI and MR-CCSD calculations were performed using the Quantum Package. ${ }^{37}$

\section{A. Single-bond breakings}

The treatment of the breaking of a single bond in principle requires only a CAS-SCF zero-order treatment including two electrons in two MOs. We have considered three problems of that type.

\section{Bond breaking of the $F_{2}$ molecule}

The $F_{2}$ molecule is a paradigmatic molecule since it is a case where the dynamical correlation brings a crucial contribution to the bonding. Despite the closed shell character of the wave function in the equilibrium region, the single reference Hartree-Fock (HF) solution is unbound (by $18 \mathrm{kcal} / \mathrm{mol}$ ) with respect to the restricted open shell $\mathrm{HF}$ solution of the fluorine atoms. The 2-electron in 2-MO CASSCF treatment binds the molecule by $18 \mathrm{kcal} / \mathrm{mol}$, but the experimental binding energy is much larger $(39 \mathrm{kcal} / \mathrm{mol})$. Going to a full valence CASSCF (14 electrons in $10 \mathrm{MOs}$ ) 
does not bring any improvement. The role of the dynamical correlation has been extensively studied and may be seen as a dynamic response of the lone pair electrons to the fluctuation of the electric field created by the two electrons of the $\sigma$ bond. ${ }^{42,43}$ The concept of orbital breathing has been proposed to express the fact that the orbitals of the lone pairs tend to become more diffuse on the negative center and more contracted on the positive center in the ionic valence-bond (VB) components of the CAS. These dynamic relaxation processes can only take place if one uses non-minimal basis sets.

The calculations of $\mathrm{F}_{2}$ were made in the cc-pVDZ basis set $^{44}$ keeping the $1 s$ electrons frozen, and accurate FCI estimates are taken from the work of Bytautas et al. ${ }^{45}$ Figure 1 shows an exponential convergence of the energy along the

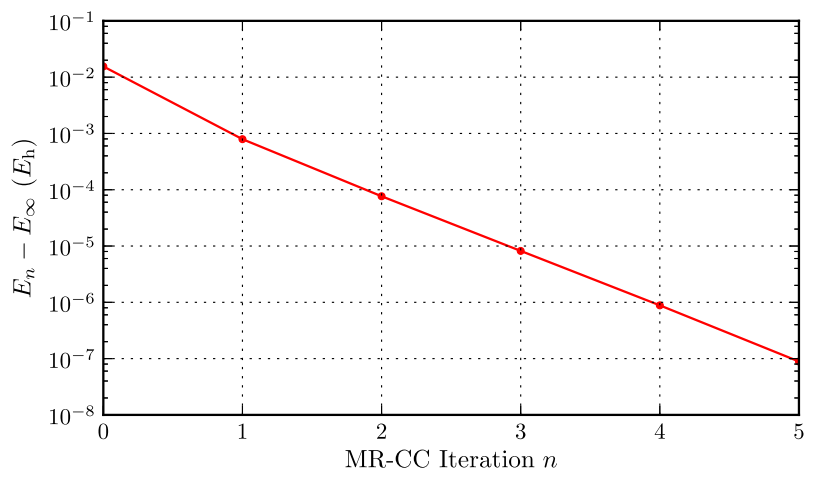

FIG. 1. $\mathrm{F}_{2}$ molecule at $R=1.45 \AA$, cc-pVDZ basis set. Convergence of the MR-CCSD energy along the iterations.

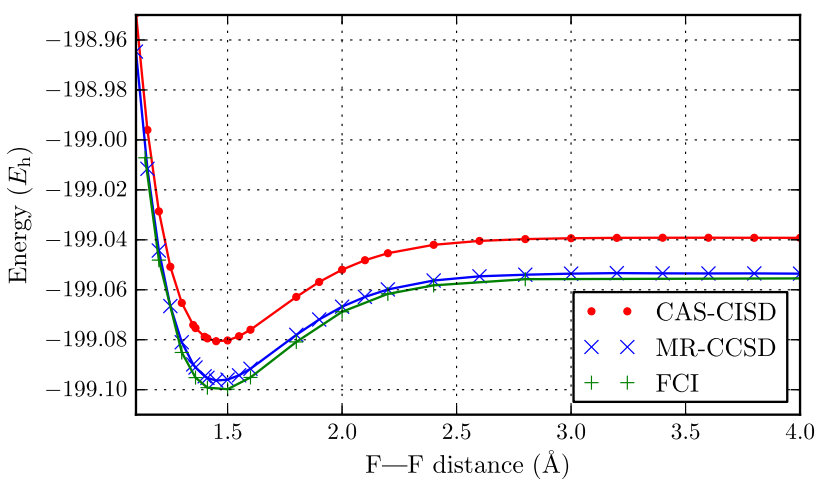

FIG. 2. Potential energy curves of the $F_{2}$ molecule, cc-pVDZ basis set.

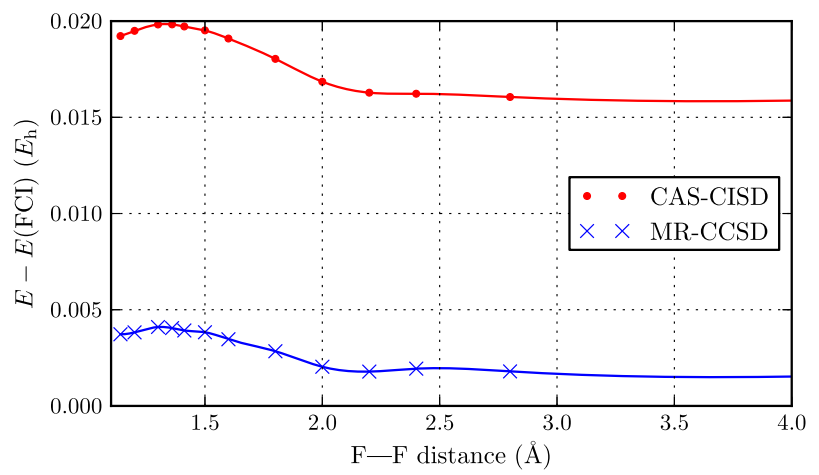

FIG. 3. $F_{2}$ molecule, cc-pVDZ basis set. Errors with respect to the FCI estimate as a function of the $\mathrm{F}-\mathrm{F}$ distance.
TABLE I. $F_{2}$ molecule, cc-pVDZ basis set. Total energies are given in $E_{\mathrm{h}}$, and the energy differences are given in $\mathrm{m} E_{\mathrm{h}}$.

\begin{tabular}{lccc}
\hline \hline$R(\AA)$ & $E_{\mathrm{CAS}-\mathrm{CISD}}-E_{\mathrm{FCI}}$ & $E_{\mathrm{MR}-\mathrm{CCSD}}-E_{\mathrm{FCI}}$ & FCI estimate \\
\hline 1.14 & 19.223 & 3.726 & -199.00718 \\
1.20 & 19.495 & 3.823 & -199.04811 \\
1.30 & 19.825 & 4.102 & -199.08510 \\
1.36 & 19.829 & 4.045 & -199.09517 \\
1.41193 & 19.721 & 3.920 & -199.09920 \\
1.50 & 19.518 & 3.830 & -199.09981 \\
1.60 & 19.094 & 3.466 & -199.09510 \\
1.80 & 18.038 & 2.843 & -199.08090 \\
2.0 & 16.850 & 2.034 & -199.06882 \\
2.2 & 16.280 & 1.783 & -199.06165 \\
2.40 & 16.225 & 1.936 & -199.05823 \\
2.80 & 16.055 & 1.794 & -199.05577 \\
8.00 & 16.241 & 1.893 & -199.05545 \\
\hline$R_{\mathrm{eq}}$ & 1.466 & 1.465 & 1.460 \\
$k_{\mathrm{eq}}$ & 0.730 & 0.739 & 0.795 \\
$D_{e}$ & 26.01 & 26.91 & 28.31 \\
$\mathrm{NPE}$ & 3.774 & 2.319 & \\
\hline \hline
\end{tabular}

MR-CC iterations. The here-reported calculation, performed in a medium size basis set, does not afford a sufficient flexibility to reach the experimental binding energy (the estimated FCI binding energy in this basis is $D_{e}=28.3 \mathrm{kcal} / \mathrm{mol}$ ). The potential energy curves and the error to FCI estimate are reported, respectively, in Figures 2 and 3, and the estimated FCI values together with the error of the MR-CCSD and CAS-CISD calculations appear in Table I. The average error, lower than $5 \mathrm{~m} E_{\mathrm{h}}$, is reduced by a factor close to 6 , and the NPE is only reduced by $40 \%$ by the MR-CCSD calculations (3.7 $\mathrm{m} E_{\mathrm{h}}$ for the CAS-CISD and $2.3 \mathrm{~m} E_{\mathrm{h}}$ for the MR-CCSD).

\section{The $C-C$ bond breaking in ethane}

This calculation is performed in the 6-31G basis set, keeping the $1 s$ electrons frozen. The geometrical parameters are given in Table II. The potential energy curves are reported in Figure 4 and the errors with respect to the FCI estimate appear in Figure 5. These data show that the error with respect to the FCI energy $\left(<3.5 \mathrm{~m} E_{\mathrm{h}}\right)$ is greatly reduced, by a factor 6 in average, with respect to the CASSDCI values. According to Table III, the NPE goes from $2.01 \mathrm{~m} E_{\mathrm{h}}$ to $1.32 \mathrm{~m} E_{\mathrm{h}}$ for, respectively, the CAS-CISD and MR-CCSD approaches. Concerning the spectroscopic constants, the impact of the CC treatment is modest but goes in the right direction.

TABLE II. Geometries used for ethane and ethylene.

\begin{tabular}{lcc}
\hline \hline Geometrical parameters & $\mathrm{C}_{2} \mathrm{H}_{6}$ & $\mathrm{C}_{2} \mathrm{H}_{4}$ \\
\hline $\mathrm{C}-\mathrm{H}$ & $1.103 \AA$ & $1.089 \AA$ \\
$\mathrm{C}-\mathrm{C}$ & $1.550 \AA$ & $1.335 \AA$ \\
$\mathrm{H}-\mathrm{C}-\mathrm{C}$ & $111.2^{\circ}$ & $120.0^{\circ}$ \\
$\mathrm{H}-\mathrm{C}-\mathrm{H}$ & $107.6^{\circ}$ & $120.0^{\circ}$ \\
$\mathrm{H}-\mathrm{C}-\mathrm{C}-\mathrm{H}$ & $180.0^{\circ}$ & $180.0^{\circ}$ \\
\hline \hline
\end{tabular}




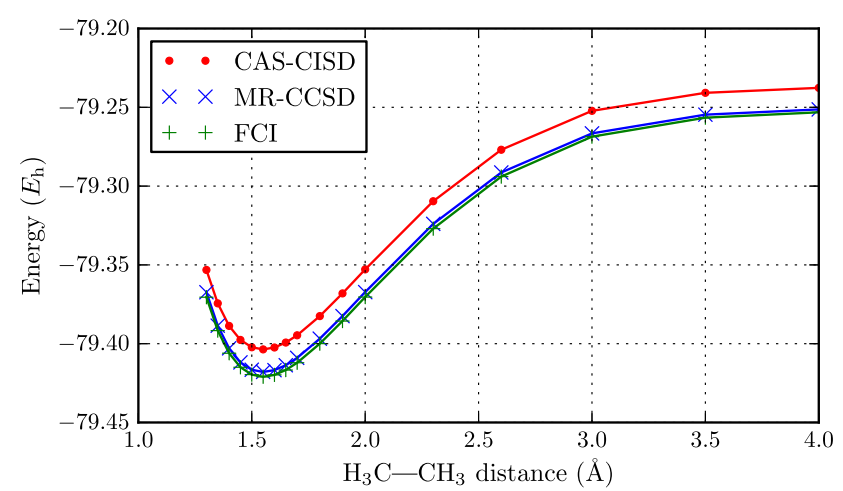

FIG. 4. Ethane molecule, 6-31G basis set. Potential energy curves along the $\mathrm{C}-\mathrm{C}$ bond stretching.

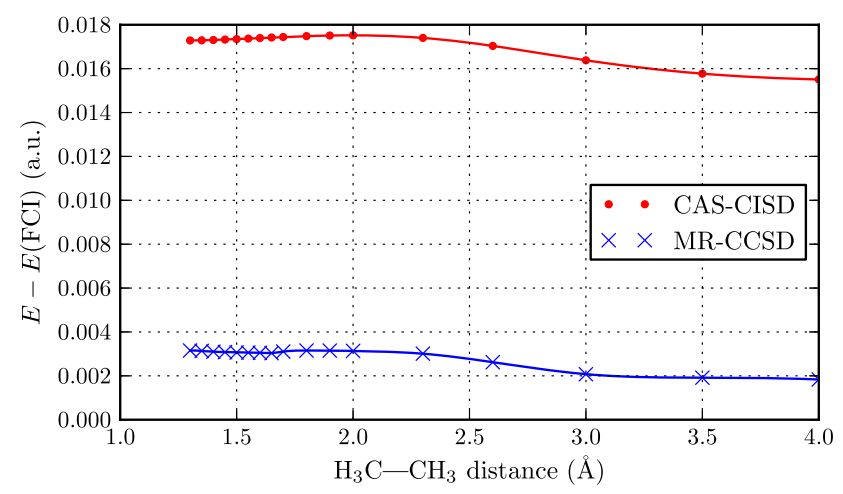

FIG. 5. Ethane molecule, 6-31G basis set. Errors with respect to the FCI estimate as a function of the $\mathrm{C}-\mathrm{C}$ distance.

TABLE III. Ethane molecule, 6-31G basis set. The FCI estimate is the CIPSI calculation. Total energies are given in $E_{\mathrm{h}}$, and the energy differences are given in $\mathrm{m} E_{\mathrm{h}}$.

\begin{tabular}{lccc}
\hline \hline$R(\AA)$ & $E_{\mathrm{CAS}-\mathrm{CISD}}-E_{\mathrm{FCI}}$ & $E_{\mathrm{MR}-\mathrm{CCSD}}-E_{\mathrm{FCI}}$ & FCI estimate \\
\hline 4.00 & 15.508 & 1.834 & -79.253166 \\
3.50 & 15.770 & 1.915 & -79.256574 \\
3.00 & 16.379 & 2.074 & -79.268617 \\
2.60 & 17.037 & 2.622 & -79.293972 \\
2.30 & 17.402 & 3.009 & -79.326999 \\
2.00 & 17.519 & 3.134 & -79.370376 \\
1.90 & 17.510 & 3.150 & -79.385598 \\
1.80 & 17.482 & 3.152 & -79.399969 \\
1.70 & 17.442 & 3.106 & -79.412107 \\
1.65 & 17.419 & 3.035 & -79.416695 \\
1.60 & 17.395 & 3.046 & -79.419813 \\
1.55 & 17.371 & 3.055 & -79.420987 \\
1.50 & 17.347 & 3.062 & -79.419613 \\
1.45 & 17.326 & 3.083 & -79.414941 \\
1.40 & 17.306 & 3.099 & -79.406030 \\
1.35 & 17.291 & 3.135 & -79.391701 \\
1.30 & 17.284 & 3.153 & -79.370480 \\
\hline$R_{e q}$ & 1.549 & 1.550 & 1.550 \\
$k_{\mathrm{eq}}$ & 1.018 & 1.017 & 1.015 \\
$D_{\mathrm{e}}$ & 104.52 & 104.99 & 105.75 \\
$\mathrm{NPE}$ & 2.011 & 1.319 & \\
\hline \hline & & & \\
\hline
\end{tabular}

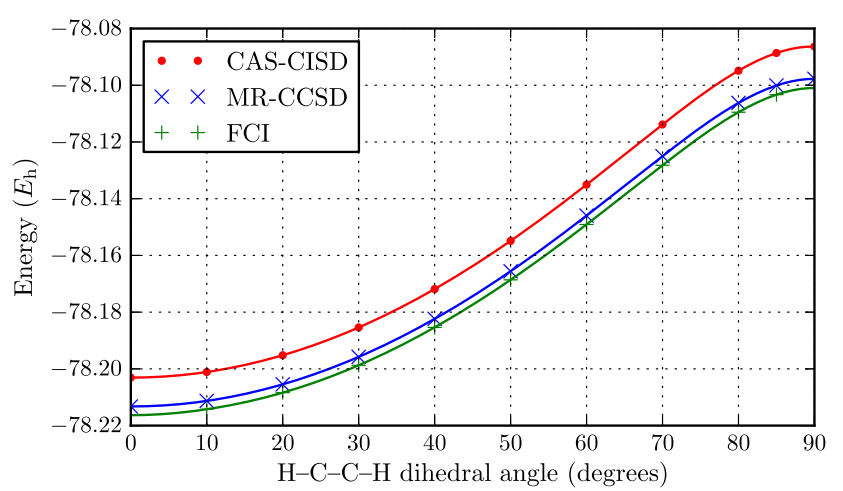

FIG. 6. Ethylene molecule, 6-31G basis set. Energy as a function of the rotation around the $\mathrm{C}-\mathrm{C}$ bond.

\section{The rotation of the ethylene molecule around its $C-C$ bond}

This twisting breaks the $\pi$ bond. The calculation is performed in the 6-31G basis set at the geometry given in Table II, keeping the $1 s$ electrons frozen. The occupied MOs in the inactive space involve 10 electrons, and despite the modest size of the basis set, one may expect a significant size-consistence defect of the CAS-CISD results, since they miss the repeatability of inactive double excitations on the $\mathrm{SD}$ determinants. The potential energy curve along the angle of rotation is reported in Figure 6 and the error to the FCI estimate is reported in Figure 7. From these data, it appears that the global shape of the potential energy curve obtained using the CC treatment is more parallel to the $\mathrm{FCI}$ curve than using the CAS-CISD approach. From Table IV, one observes that the error with respect to the FCI estimate (about $3 \mathrm{~m} E_{\mathrm{h}}$ ) is reduced by a factor of 6 when going from CAS-CISD to MR-CCSD. Also, the NPE is also reduced from $1.6 \mathrm{~m} E_{\mathrm{h}}$ to $0.3 \mathrm{~m} E_{\mathrm{h}}$.

\section{B. Insertion of $\mathrm{Be}$ in $\mathrm{H}_{2}$}

The $\mathrm{Be}+\mathrm{H}_{2}$ system is a popular benchmark for MR-CC methods. ${ }^{46-54}$ This chemical reaction is somewhat more complex than the single bond breaking and the use of $\operatorname{CAS}(2,2)$ is questionable but this reference space has been used here for comparison with the literature. The geometries

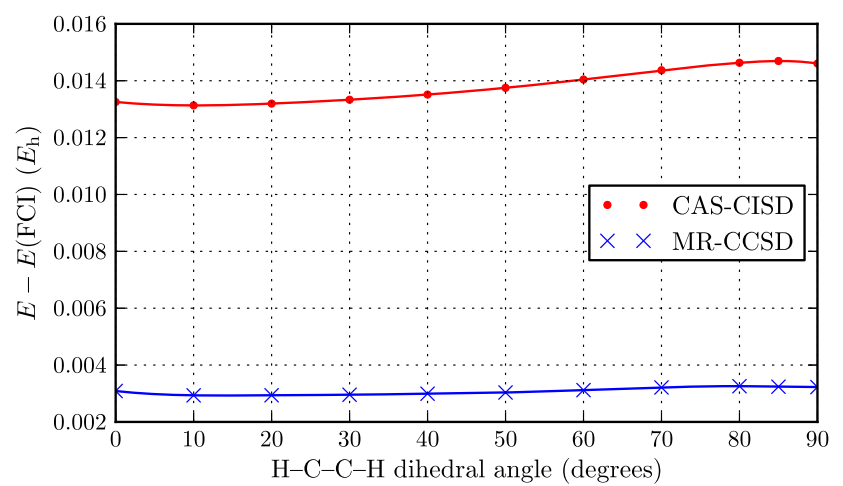

FIG. 7. Ethylene molecule, 6-31G basis set. Errors with respect to the FCI estimate as a function of the rotation around the $\mathrm{C}-\mathrm{C}$ bond. 
TABLE IV. Rotation of the ethylene molecule, 6-31G basis set. The FCI estimate is the CIPSI calculation. Total energies are given in $E_{\mathrm{h}}$, and the energy differences are given in $\mathrm{m} E_{\mathrm{h}}$.

\begin{tabular}{lccc}
\hline \hline Angles $(\mathrm{deg})$ & $E_{\mathrm{CAS}-\mathrm{CISD}}-E_{\mathrm{FCI}}$ & $E_{\mathrm{MR}-\mathrm{CCSD}}-E_{\mathrm{FCI}}$ & FCI estimate \\
\hline 0 & 13.255 & 2.935 & -78.216340 \\
10 & 13.132 & 2.935 & -78.214241 \\
20 & 13.196 & 2.938 & -78.208391 \\
30 & 13.331 & 2.955 & -78.198732 \\
40 & 13.513 & 2.991 & -78.185373 \\
50 & 13.750 & 3.035 & -78.168619 \\
60 & 14.043 & 3.120 & -78.149094 \\
70 & 14.368 & 3.212 & -78.128205 \\
80 & 14.631 & 3.258 & -78.109498 \\
85 & 14.694 & 3.237 & -78.103326 \\
90 & 14.605 & 3.227 & -78.100966 \\
\hline $\mathrm{NPE}$ & 1.562 & 0.323 & \\
\hline \hline
\end{tabular}

are given by the relation

$$
z=2.54-0.46 x \quad \text { (a.u.) }
$$

where the berylium atom is at the origin and the hydrogen atoms at the coordinate $(x, 0, \pm z)$. As in previous calculations, the $1 s$ electrons are kept frozen. The two active orbitals result from the $\operatorname{CASSCF}(2,2)$ optimization starting with the HOMO and LUMO orbitals of the RHF calculation. The results are given in Table V. From this table, it appears that the MR-CCSD reduces both the absolute error to the FCI energy and the NPE by a factor between 2 and 3, with respect to the CAS-CISD calculations. The NPE with our proposal is of $1.297 \mathrm{~m} E_{\mathrm{h}}$ in the cc-pVDZ basis set. This value compares

TABLE V. $\mathrm{BeH}_{2}$ molecule, cc-pVDZ basis set. Total energies are given in $E_{\mathrm{h}}$, and the energy differences are given in $\mathrm{m} E_{\mathrm{h}}$.

\begin{tabular}{|c|c|c|c|}
\hline$X$ (a.u.) & $E_{\mathrm{CAS}-\mathrm{CISD}}-E_{\mathrm{FCI}}$ & $E_{\mathrm{MR}-\mathrm{CCSD}}-E_{\mathrm{FCI}}$ & FCI estimate \\
\hline 0 & 1.212 & 0.470 & -15.835475 \\
\hline 0.2 & 1.202 & 0.471 & -15.833837 \\
\hline 0.4 & 1.204 & 0.468 & -15.828821 \\
\hline 0.6 & 1.217 & 0.433 & -15.821276 \\
\hline 0.8 & 1.245 & 0.458 & -15.812169 \\
\hline 1 & 1.291 & 0.481 & -15.802262 \\
\hline 1.2 & 1.358 & 0.518 & -15.791845 \\
\hline 1.4 & 1.450 & 0.438 & -15.780681 \\
\hline 1.6 & 1.567 & 0.615 & -15.768174 \\
\hline 1.8 & 1.679 & 0.655 & -15.753649 \\
\hline 2 & 1.648 & 0.761 & -15.736620 \\
\hline 2.2 & 1.807 & 0.837 & -15.716964 \\
\hline 2.4 & 2.107 & 1.032 & -15.695068 \\
\hline 2.6 & 2.538 & 1.464 & -15.672378 \\
\hline 2.8 & 3.333 & 1.767 & -15.655899 \\
\hline 3 & 4.093 & 1.276 & -15.666560 \\
\hline 3.2 & 3.880 & 1.141 & -15.690150 \\
\hline 3.4 & 3.546 & 0.958 & -15.714088 \\
\hline 3.6 & 2.623 & 0.983 & -15.735526 \\
\hline 3.8 & 2.492 & 0.786 & -15.752272 \\
\hline 4 & 2.681 & 0.569 & -15.760567 \\
\hline NPE & 2.891 & 1.297 & \\
\hline
\end{tabular}

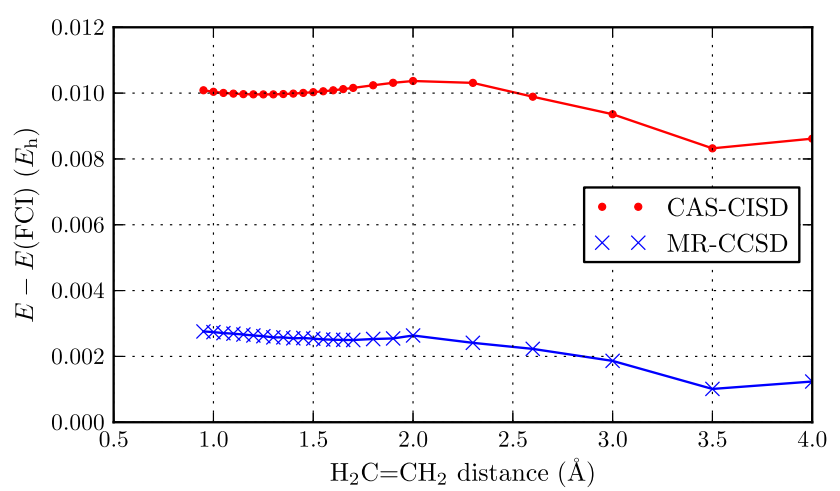

FIG. 8. Potential energy curves of the ethylene molecule, 6-31G basis set. Errors with respect to the FCI estimate as a function of the $\mathrm{C}-\mathrm{C}$ distance.

favourably with the values reported in the literature using a smaller basis set, namely, the Mk-MRCCSD $\left(2.238 \mathrm{~m} E_{\mathrm{h}}{ }^{49,53}\right)$, which falls to $1.355 \mathrm{~m} E_{\mathrm{h}}$ after a perturbative inclusion of the triple excitations, ${ }^{53} 1.663 \mathrm{~m} E_{\mathrm{h}}$ of the exponential wave function Ansatz of Hanrath. ${ }^{51}$

\section{Two-bond breakings}

Three systems have been treated using a CAS with four electrons in 4 active MOs. Two of them concern the simultaneous breaking of two bonds.

\section{Breaking of the $C=C$ double bond of ethylene}

The dissociation of the ethylene molecule by breaking the double bond was studied in the 6-31G basis set, with the geometry given in Table II. We report the error $\left(<3 \mathrm{~m} E_{\mathrm{h}}\right)$ with respect to the FCI estimate in Figure 8. The corresponding values appear in Table VI. Again, the error to estimated FCI energy is reduced by a factor of 4 , but the NPE is reduced only by $20 \%$ with the $\mathrm{CC}$ treatment.

\section{Two-bond breaking in $\mathrm{H}_{2} \mathrm{O}$}

This is a rather well known test problem for MR-CC methods. The calculation is done with the cc-pVDZ basis set at five different geometries obtained from the equilibrium geometry $\left(R_{e}=1.84345 \AA\right.$ and $\left.\angle_{\mathrm{HOH}}=110.6^{\circ}\right)$, in order to compare with the values of the literature. ${ }^{40}$ The results appear in Table VII. The benefit of the MR-CCSD with respect to the CAS-CISD treatment is significant: the maximum error is $1.4 \mathrm{~m} E_{\mathrm{h}}$, better than the $6.4 \mathrm{~m} E_{\mathrm{h}}$ given by the Mk-MR$\mathrm{CC}$ treatment. This improvement may be due to the hereproposed treatment of the amplitudes responsible for potential divergences. The NPE goes from $2 \mathrm{~m} E_{\mathrm{h}}$ to $0.7 \mathrm{~m} E_{\mathrm{h}}$ when the $\mathrm{CC}$ treatment is applied.

\section{Triple-bond breaking in $\mathbf{N}_{\mathbf{2}}$}

The dissociation of $\mathrm{N}_{2}$ is a popular benchmark for multireference methods. It requires a 6-electron in 6-MO CAS. Calculations were done with the cc-pVDZ basis set at the six inter-atomic distances taken from Ref. 40. The results are reported in Table VIII. 
TABLE VI. Dissociation of the ethylene molecule, 6-31G basis set. The FCI estimate is the CIPSI calculation. Total energies are given in $E_{\mathrm{h}}$, and the energy differences are given in $\mathrm{m} E_{\mathrm{h}}$.

\begin{tabular}{|c|c|c|c|}
\hline$R(\AA)$ & $E_{\mathrm{CAS}-\mathrm{CISD}}-E_{\mathrm{FCI}}$ & $E_{\mathrm{MR}-\mathrm{CCSD}}-E_{\mathrm{FCI}}$ & FCI estimate \\
\hline 1.20 & 9.962 & 2.635 & -78.179508 \\
\hline 1.25 & 9.960 & 2.614 & -78.200805 \\
\hline 1.30 & 9.961 & 2.578 & -78.212507 \\
\hline 1.35 & 9.971 & 2.578 & -78.216869 \\
\hline 1.40 & 9.985 & 2.549 & -78.215666 \\
\hline 1.45 & 10.006 & 2.558 & -78.210313 \\
\hline 1.50 & 10.028 & 2.539 & -78.201918 \\
\hline 1.55 & 10.055 & 2.517 & -78.191365 \\
\hline 1.60 & 10.085 & 2.507 & -78.179345 \\
\hline 1.65 & 10.121 & 2.500 & -78.166404 \\
\hline 1.70 & 10.158 & 2.498 & -78.152957 \\
\hline 1.80 & 10.238 & 2.528 & -78.125775 \\
\hline 1.90 & 10.313 & 2.545 & -78.099562 \\
\hline 2.00 & 10.368 & 2.635 & -78.075294 \\
\hline 2.30 & 10.310 & 2.411 & -78.017944 \\
\hline 2.60 & 9.890 & 2.228 & -77.983952 \\
\hline 3.00 & 9.358 & 1.863 & -77.964220 \\
\hline 3.50 & 8.321 & 1.011 & -77.956421 \\
\hline 4.00 & 8.616 & 1.237 & -77.955127 \\
\hline$R_{\text {eq }}$ & 1.362 & 1.362 & 1.362 \\
\hline$k_{\mathrm{eq}}$ & 2.043 & 2.039 & 2.042 \\
\hline$D_{e}$ & 163.48 & 163.54 & 164.47 \\
\hline NPE & 2.047 & 1.624 & \\
\hline
\end{tabular}

As the number of inactive electrons is rather small, the CAS-CISD results are already quite good: the NPE is $1.088 \mathrm{~m} E_{\mathrm{h}}$ and the error is in the order of 8-9 $\mathrm{m} E_{\mathrm{h}}$. The CC treatment reduces the mean error to $\sim 2.5 \mathrm{~m} E_{\mathrm{h}}$ and the NPE decreases to $0.45 \mathrm{~m} E_{\mathrm{h}}$. These results are much better than those of the Mk-MR-CCSD method and of a quality similar to that obtained by the Single Reference-Multi-Reference Coupled Cluster (SR-MR-CC) method of Oliphant and Adamowicz. ${ }^{30}$

\section{PROPERTIES}

\section{A. Internal decontraction}

The method is internally decontracted. The coefficients of the references as well as those of the singles and doubles change along the iterations. If the reference space is a valence CAS, treating the non-dynamical correlation effects, the method takes care of the impact of the dynamical correlation on the non-dynamical part. The phenomenon is especially important in magnetic systems where the dynamical charge polarization effects increase dramatically the weight of the ionic valence bond components, diminishing severely the effective energy of these components. ${ }^{43}$ This effect is already present in the CAS-CISD calculation but the MR-CCSD treatment eliminates the size consistency defect and slightly improves the quality of the projection of the wave function on the CAS.

TABLE VII. Symmetric dissociation of the water molecule, cc-pVDZ basis set. The FCI total energy ${ }^{55}$ is given in $E_{\mathrm{h}}$, and the deviations to this reference are given in $\mathrm{m} E_{\mathrm{h}}$. Comparison with Mukherjee's state specific MR-CC values ( $\left.E_{\mathrm{Mk}-\mathrm{MR}-\mathrm{CCSD}}-E_{\mathrm{FCI}}\right)$ obtained from Ref. 40.

\begin{tabular}{lcccc}
\hline \hline$R\left(R_{e}\right)$ & $E_{\mathrm{CAS}-\mathrm{CISD}}-E_{\mathrm{FCI}}$ & $E_{\mathrm{Mk}-\mathrm{MR}-\mathrm{CCSD}}-E_{\mathrm{FCI}}$ & $E_{\mathrm{MR}-\mathrm{CCSD}}-E_{\mathrm{FCI}}$ & FCI \\
\hline $1 R_{e}$ & 4.923 & 2.909 & 1.407 & -76.241860 \\
$1.5 R_{e}$ & 4.674 & 4.817 & 1.248 & -76.072348 \\
$2.0 R_{e}$ & 3.665 & 6.485 & 0.855 & -75.951665 \\
$2.5 R_{e}$ & 3.097 & 5.672 & 0.763 & -75.917991 \\
$3.0 R_{e}$ & 2.959 & 3.987 & 0.845 & -75.911946 \\
\hline $\mathrm{NPE}$ & 1.964 & 3.576 & 0.644 & \\
\hline \hline
\end{tabular}

TABLE VIII. Dissociation of the $\mathrm{N}_{2}$ molecule, cc-pVDZ basis set. The FCI total energy ${ }^{56}$ is given in $E_{\mathrm{h}}$, and the deviations to this reference are given in $\mathrm{m} E_{\mathrm{h}}$. Comparison with Mukherjee's state specific MR-CC values $\left(E_{\mathrm{Mk}-\mathrm{MR}-\mathrm{CCSD}}-E_{\mathrm{FCI}}\right)$ and SR-MR-CCSD values $\left(E_{\mathrm{SR}-\mathrm{MR}-\mathrm{CCSD}}-E_{\mathrm{FCI}}\right)$ obtained from Ref. 40.

\begin{tabular}{|c|c|c|c|c|c|}
\hline$R\left(a_{0}\right)$ & $E_{\mathrm{CAS}-\mathrm{CISD}}-E_{\mathrm{FCI}}$ & $E_{\mathrm{Mk}-\mathrm{MR}-\mathrm{CCSD}}-E_{\mathrm{FCI}}$ & $E_{\mathrm{SR}-\mathrm{MR}-\mathrm{CCSD}}-E_{\mathrm{FCI}}$ & $E_{\mathrm{MR}-\mathrm{CCSD}}-E_{\mathrm{FCI}}$ & FCI \\
\hline 2.118 & 8.320 & 6.916 & 1.515 & 2.403 & -109.278339 \\
\hline 2.4 & 8.774 & 8.785 & 1.528 & 2.472 & -109.238397 \\
\hline 2.7 & 9.163 & 11.071 & 1.618 & 2.589 & -109.160305 \\
\hline 3.0 & 9.408 & 13.506 & 1.789 & 2.671 & -109.086209 \\
\hline 3.6 & 9.205 & 18.413 & 2.187 & 2.485 & -108.994906 \\
\hline 4.2 & 8.485 & 18.950 & 2.246 & 2.220 & -108.966950 \\
\hline NPE & 1.088 & 12.033 & 0.731 & 0.451 & \\
\hline
\end{tabular}




\section{B. Size consistence and separability}

The size consistence and strict separability properties are crucial issues of multi-reference methods. ${ }^{57}$ The rigorous formulation of the method does not introduce any unlinked diagram and is therefore size-consistent. However, the fifthorder approximation introduced by simply considering the products of $\tilde{d}_{I k}^{m}$ (see Section II E) breaks the strict separability. The strict separability requires that in the splitting into two subsystems A and B, the active and inactive MOs are localized on one of the two subsystems A or B. Actually, as occurs for the Mk-MR-CC formalism, the method is not invariant with respect to the unitary transformation of the MOs in their class (inactive occupied, active, inactive virtual). This dependence will be studied in a future work, but the error to FCI being small, we do not expect a strong dependence on the MO definition. As was shown in the study of bond breakings, the asymptotic size-consistency error (which is demonstrated to be zero when localized MOs are used) is negligible in a basis of symmetry-adapted MOs.

Numerical tests were performed in the cc-pVDZ basis set on a $\mathrm{Li}+\mathrm{F}$ problem at $R=16 \AA$ using a 2-electron in 2-orbital CAS-CISD and MR-CCSD. At the CAS-CISD level, the nonadditivity error is $6 \times 10^{-5} E_{\mathrm{h}}$, and it is reduced by a factor of 10 at the MR-CCSD level. The $\mathrm{Be}+\mathrm{H}_{2}$ system was also studied for $R=20 \AA$ using both a CAS-CISD with the two electrons of the $\mathrm{Be}$ atom in the $2 s$ and $2 p$ orbitals of $\mathrm{Be}$, and also adding to the CAS the 2 electrons of $\mathrm{H}_{2}$ in the bonding and anti-bonding MOs of the molecule. The CAS-CISD energies deviate from additivity by, respectively, $1.8 \mathrm{~m} E_{\mathrm{h}}$ and $0.9 \mathrm{~m} E_{\mathrm{h}}$. The additivity is recovered by the MR-CCSD at a precision $5 \times 10^{-6} E_{\mathrm{h}}$. The total energies are given in Table IX.

This small deviation is due to the approximation introduced in Section II E $\left(d_{I i}^{m} \approx \tilde{d}_{I i}^{m}\right)$ : in the case where the interaction $H_{I i}$ is zero, our approximation of the amplitudes, based on this matrix element, cancels the corresponding $d_{I i}^{m}$, while the amplitudes of the single excitations contained in the $\tilde{d}_{I i}^{m}$ may be non-zero. This is the case at the separation limit into two free radicals $A$. and $B$, and the single excitations $s_{I, \hat{T}_{i} \rightarrow r_{A} I}$ on $A$ and $s_{I, \hat{T}_{\bar{J}_{B} \rightarrow \bar{s}_{B}} I}$ on $B$ may be non-zero (as will be the case for instance for the spin polarization of the cores), while $\left\langle I|H| a_{r_{A}}^{\dagger} a_{\bar{s}_{B}}^{\dagger} a_{\bar{j}_{B}} a_{i_{A}} I\right\rangle=0$, since it is a dispersion type double excitation. Our approximation omits the action of the

TABLE IX. Total energies (cc-pVDZ) for the numerical check of the separability of $\mathrm{Li} \cdots \mathrm{F}$ and $\mathrm{Be} \cdots \mathrm{H}_{2}$. (The $\mathrm{H}-\mathrm{H}$ distance was set to $1 \AA$ to magnify the correlation on $\mathrm{H}_{2}$.)

\begin{tabular}{lrr}
\hline & \multicolumn{1}{c}{ CAS-CISD } & \multicolumn{1}{c}{ MR-CCSD } \\
\hline $\mathrm{Li}$ & -7.433463 & -7.433463 \\
$\mathrm{~F}$ & -99.528807 & -99.531870 \\
$\mathrm{Li}+\mathrm{F}$ & -106.962270 & -106.965334 \\
$\mathrm{Li} \cdots \mathrm{F}$ & -106.962209 & -106.965342 \\
\hline $\mathrm{Be}$ & -14.618519 & -14.618536 \\
$\mathrm{H} 2$ & -1.140073 & -1.140073 \\
$\mathrm{Be}+\mathrm{H}_{2}$ & -15.758592 & -15.758609 \\
$\mathrm{Be} \cdots \mathrm{H}_{2} \mathrm{CAS}(2,4)$ & -15.756791 & -15.758604 \\
$\mathrm{Be} \cdots \mathrm{H}_{2} \mathrm{CAS}(4,6)$ & -15.757693 & -15.758605 \\
\hline \hline
\end{tabular}

products $s_{I, \hat{T}_{i_{A} \rightarrow r_{A} I} I} \times s_{I, \hat{T}_{\bar{j}_{B} \rightarrow \bar{s}_{B}} I}$ on the singles and doubles, the dominant part concerning intra-system excitations. In our opinion, the computational benefit of the neglect of some high powers of the single excitations legitimates this approximation in the MR-CC treatment.

\section{Eigenfunction of $\hat{\boldsymbol{S}}^{2}$}

The here-proposed method does not provide an eigenfunction of $\hat{S}^{2}$ as we consider only the determinants that are connected by an application of $\hat{H}$ to the determinants belonging to the CAS. This treatment does not include higher excitations which will generate the full space associated with a given space part. Along all the performed calculations on singlet states, the order of magnitude of the expectation value of $\hat{S}^{2}$ calculated on both the CAS-CISD and the projected MR-CCSD wave functions never exceeded $10^{-3}$. A solution working with the same restricted space but providing a strict eigenfunction of $\hat{S}^{2}$ is presented in Sec. VI.

\section{PROSPECTS}

\section{A. Computational cost}

The method is extremely flexible, either on the choice of the reference space and/or of the excitations from it. As the theory is determinant based, one can take advantage of this flexibility to realize a CIPSI-like selection of the dominant contributions of both the references and the single and double excitations. Further works will investigate the various possibilities such as the combination of MR-CC with perturbation theory in order to target more realistic systems.

\section{B. Excited states}

The method is applicable to excited states using several approaches. The formalism being state specific, the dressing technique of the CI matrix can be applied to any state dominated by the reference determinants, as long as a state following procedure is applied. For states belonging to the same symmetry, the resulting eigenvectors will not be strictly orthogonal but might be orthogonalized a posteriori. Another possibility consists of a state average procedure where the amplitudes are obtained from the values of the quantities $\lambda_{i}$ averaged over all desired eigenstates,

$$
\lambda_{i}=\frac{\sum_{m} \lambda_{i}^{m}\left(c_{i}^{m}\right)^{2}}{\sum_{m}\left(c_{i}^{m}\right)^{2}} .
$$

If one refers to the perturbative expression of the first order coefficients,

$$
\lambda_{i}^{m} \approx \frac{1}{E_{0}^{m}-\langle i|\hat{H}| i\rangle},
$$

this approximation should be relevant when the states are close enough in energy.

A recent paper $^{58}$ has proposed a generalization of this approach to the simultaneous treatment of several states of the same symmetry. The basic ideas are the same, except for the fact that the extraction of the amplitudes is more complex. 
The method requires to partition the reference space into a main and an intermediate model spaces, in the spirit of the intermediate Hamiltonian formalism. ${ }^{15}$ This proposal will be tested in a further work.

\section{Spin multiplicity conservation}

As already mentioned the space of the singles and doubles is limited here to the determinants which interact with at least one of the references. If the references already have several open shells, this condition drastically reduces the size of the CASSD with respect to the space involving all the determinants of the configurations which interact with the references through at least one of their determinants. The price to pay is some minor deviation of the eigenvector from an $\hat{S}^{2}$ eigenvector. If one wants to ensure this property, one may follow one of two following routes. If the one calls $i_{1}, i_{2}, \ldots, i_{k}, \ldots$ the determinants which belong to the same space configuration and interact with at least one reference, they may receive various values of the effective inverse energy $\lambda_{i k}^{m}$. If one defines a unique value $\lambda_{i}^{m}$ for all the determinants of the configuration, for instance, the mean value of the $\lambda_{i k}^{m}$, s,

$$
\lambda_{i}^{m}=\frac{\sum_{k} \lambda_{i k}^{m}\left(c_{i k}^{m}\right)^{2}}{\sum_{k}\left(c_{i k}^{m}\right)^{2}},
$$

the vector

$$
|i\rangle=\lambda_{i}^{m} \sum_{k}\left\langle\Psi_{0}^{m}|\hat{H}| i_{k}\right\rangle\left|i_{k}\right\rangle
$$

is an eigenfunction of $\hat{S}^{2}$. This is due to the fact that $\hat{S}^{2}$ does not change the space part of the determinants and that it commutes with $\hat{H}$. Consequently, the excitation operator

$$
\hat{T}_{I i} d_{I i}^{m}=\lambda_{i}^{m} \sum_{k}\left\langle I|\hat{H}| i_{k}\right\rangle \hat{T}_{I k}
$$

commutes with $\hat{S}^{2}$. The products of such operators, which have been used to generate the coefficients of the triples and quadruples, also commute with $\hat{S}^{2}$, and the CC process should keep the exact spin multiplicity.

The other way to ensure that property, which is by far more expensive, would consist of starting from the complete CASSD, including the determinants $i_{h}^{\prime}$ which do not interact with the references. The eigenvector is then an eigenfunction of $\hat{S}^{2}$. A parentage must be defined between the references and the determinants $i^{\prime}$, possibly by considering the interactions between the determinants of type $i^{\prime}$ and the determinants of type $i$. But this strategy would introduce many-body (more than 2-body) operators, which would make the algorithm much more complex and costly.

\section{Possible extensions}

The method is extremely flexible. One may, for instance, define a subset of inactive MOs (occupied and virtual) which have the major contribution to the dynamical correlation, and divide the CISD subspace into two parts, the "internal" excitations involving only the MOs of the selected subset would define a selected fraction of the CAS-CISD, and the effect of the triples and quadruples issued from the action of the "internal" double excitations on the selected CAS-CISD would lead to the dressing of the interactions between the references and the selected singles and doubles, while the rest of the CASCISD matrix would be untouched. This would correct the major size-inconsistency defect of the CAS-CISD treatment and introduce the major fourth-order contributions. Combinations of MR-CC and perturbations will be considered as well.

In order to reduce the computation time, the purely inactive double excitations, which are the most numerous, may be treated in a specific manner. Let us consider a composite system $A \cdots B$ where the active space is localized on $A$, and where the interactions between $A$ and $B$ vanish, and suppose that one is interested in the spectrum of $A, B$ being in its ground state $\left|0_{B}\right\rangle$. The model space determinants have the form $\left|I_{A}^{\prime}\right\rangle=\left|I_{A} 0_{B}\right\rangle$. The effect of the double excitations on $B$ should be a simple shift of the energies. This is the weak separability condition. It is not satisfied at the CAS-CISD level, due to spurious normalization effects. The inactive double excitations $\hat{T}_{l_{B}}$ on $B$ are possible on all references $\left|I_{A}\right\rangle$ and the coefficient of the resulting determinant should be

$$
c_{\hat{T}_{l_{B}} I_{A}}=c_{l_{B}} C_{I_{A}}
$$

where $c_{l_{B}}$ is the coefficient relative to the double excitation $\hat{T}_{l_{B}}$ on $B$. The correlation energy brought by the double excitation on $B$ is

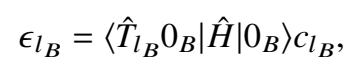

and this effect may be introduced as an energy shift on the diagonal energies of all the determinants of the CASCISD matrix, as occurs for the $(\mathrm{SC})^{2}$ dressing in the single reference context. At convergence this dressing ensures the weak separability property. We suggest to extend this remark to all the inactive double excitations whatever the interaction between the active and inactive orbitals. Considering the CASCISD, one may define a first evaluation of the contribution of any inactive double excitation as the averaged contributions of the inactive double excitation $\hat{T}_{l}$ on the various references,

$$
\epsilon_{l}=\frac{\sum_{I}\left\langle\hat{T}_{l} I|\hat{H}| I\right\rangle c_{\hat{T}_{l} I} C_{I}}{\sum_{I} C_{I}{ }^{2}} .
$$

Then the effect of all the inactive double excitations should be summed and introduced as a shift of the diagonal energies of all the SD determinants in the dressed CAS-CISD matrix. The inactive double excitations should no longer be considered in the generation of the triples and quadruples determinants $|\alpha\rangle$ in the MR-CC treatment.

Another extension will concern the Difference Dedicated Configuration Interaction (DDCI), which is a standard method in the treatment of magnetic systems. ${ }^{59}$ The method is based on a CAS and eliminates the purely inactive double excitations, which are the most numerous and do not contribute (at least to the second-order) to the vertical energy differences. Due to this restriction, the error of size consistence is reduced but it exists and may be non-negligible when the number of active electrons increases. It is perfectly possible to couple-clusterize the DDCI method by repeating the effect of the semi-active excitations only on the determinants appearing in the DDCI space. 


\section{CONCLUSION}

This work shows the relevance of a solution previously proposed to the problem of the multiple parentage faced by all Multi-reference treatments, as soon as the number of targeted vectors is lower than the number of references. The proposed MR-CC algorithm is simple. It only introduces two-body excitation operators and the number of amplitudes to be determined is reduced to the very minimum. It proceeds through an iterative dressing of the MR-CI matrix, formulated in terms of a standard eigenvalue equation. It is parallelizable in the most expensive step (the generation of the coefficients of the triples and quadruples). It is entirely decontracted and may be applied to excited states. A convenient simplification introducing a fifth-order deviation on the energy was introduced. For the list of benchmark studies we have performed, the results are extremely encouraging. The present version is state-specific but the principles of extension to a multi-root version have been formulated. ${ }^{58}$ This work actually opens into several directions, which have to be explored in the future. The reduction of the computational cost might be done using several approximations involving the selection of the references and/or singles and doubles according to various criteria. Furthermore, the excited states may be treated either in the present state-specific formalism or using its multi-state generalization.

On a different perspective, the multiple parentage problem, which was faced here with the purpose of building a logically consistent computational tool to go in the direction of the exact solution, also concerns the building of rational valence-only effective Hamiltonians. In such an approach, the idea is to map the information coming from a sophisticated treatment into a minimal effective Hamiltonian, the parameters of which should be as physically meaningful as possible. ${ }^{60} \mathrm{We}$ believe that the solution we proposed to the multiple parentage problem offers a rational solution to this reduction of information. This remark illustrates the intrinsic link between the two main tasks of quantum chemistry, namely, the production of physically grounded interpretative models on one hand and the conception of rigorous computational tools.

${ }^{1}$ J. Goldstone, Proc. R. Soc. A 239, 267 (1957).

${ }^{2}$ H. P. Kelly and M. A. Sessler, Phys. Rev. 132, 2091 (1963).

${ }^{3}$ H. P. Kelly, Phys. Rev. 134, A1450 (1964).

${ }^{4}$ W. Meyer, Int. J. Quantum Chem. 5, 341 (1971).

${ }^{5}$ W. Meyer, J. Chem. Phys. 58, 1017 (1973).

${ }^{6}$ W. Meyer, Theor. Chim. Acta 35, 277 (1974).

${ }^{7}$ J. P. Daudey, J. L. Heully, and J. P. Malrieu, J. Chem. Phys. 99, 1240 (1993).

${ }^{8}$ F. Coester, Nucl. Phys. 7, 421 (1958).

${ }^{9}$ F. Coester and H. Kümmel, Nucl. Phys. 17, 477 (1960).

${ }^{10}$ J. Čiźěk, J. Chem. Phys. 45, 4256 (1966).

${ }^{11}$ R. J. Bartlett, J. Watts, S. Kucharski, and J. Noga, Chem. Phys. Lett. 165, 513 (1990).

${ }^{12}$ B. H. Brandow, Rev. Mod. Phys. 39, 771 (1967).

${ }^{13}$ B. Huron, P. Rancurel, and J. P. Malrieu, J. Chem. Phys. 58, 5745 (1973).

${ }^{14}$ S. Evangelisti, J. P. Daudey, and J. P. Malrieu, Chem. Phys. 75, 91 (1983).

${ }^{15}$ J. P. Malrieu, P. Durand, and J. P. Daudey, J. Phys. A: Math. Gen. 18, 809 (1985).

${ }^{16}$ B. Kirtman, J. Chem. Phys. 75, 798 (1981).
${ }^{17}$ J. L. Heully, J. P. Malrieu, and A. Zaitsevskii, J. Chem. Phys. 105, 6887 (1996).

${ }^{18}$ K. Andersson, P. Malmqvist, B. O. Roos, A. J. Sadlej, and K. Wolinski, J. Phys. Chem. 94, 5483 (1990).

${ }^{19}$ K. Andersson, P. Malmqvist, and B. O. Roos, J. Chem. Phys. 96, 1218 (1992).

${ }^{20}$ C. Angeli, R. Cimiraglia, S. Evangelisti, T. Leininger, and J. P. Malrieu, J. Chem. Phys. 114, 10252 (2001).

${ }^{21}$ C. Angeli, R. Cimiraglia, and J. P. Malrieu, Chem. Phys. Lett. 350, 297 (2001).

${ }^{22}$ C. Angeli, R. Cimiraglia, and J. P. Malrieu, J. Chem. Phys. 117, 9138 (2002).

${ }^{23}$ K. G. Dyall, J. Chem. Phys. 102, 4909 (1995).

${ }^{24}$ P. Celani and H. J. Werner, J. Chem. Phys. 112, 5546 (2000).

${ }^{25}$ P. Ghosh, S. Chattopadhyay, D. Jana, and D. Mukherjee, Int. J. Mol. Sci. 3, 733 (2002).

${ }^{26}$ U. S. Mahapatra, B. Datta, and D. Mukherjee, Chem. Phys. Lett. 299, 42 (1999).

${ }^{27}$ A. Sen, S. Sen, P. K. Samanta, and D. Mukherjee, J. Comput. Chem. 36, 670 (2015).

${ }^{28}$ P. G. Szalay, T. Müller, G. Gidofalvi, H. Lischka, and R. Shepard, Chem. Rev. 112, 108 (2012).

${ }^{29}$ D. I. Lyakh, M. Musiał, V. F. Lotrich, and R. J. Bartlett, Chem. Rev. 112, 182 (2012).

${ }^{30}$ N. Oliphant and L. Adamowicz, J. Chem. Phys. 94, 1229 (1991).

${ }^{31}$ J. Meller, J. P. Malrieu, and R. Caballol, J. Chem. Phys. 104, 4068 (1996).

${ }^{32}$ U. S. Mahapatra, B. Datta, and D. Mukherjee, Mol. Phys. 94, 157 (1998).

${ }^{33}$ J. Mášik and I. Hubač, Advances in Quantum Chemistry (Academic Press, 1998), pp. 75-104.

${ }^{34}$ I. Hubač, J. Pittner, and P. Čársky, J. Chem. Phys. 112, 8779 (2000).

${ }^{35}$ B. Jeziorski and H. J. Monkhorst, Phys. Rev. A 24, 1668 (1981).

${ }^{36}$ I. Nebot-Gil, J. Sańchez-Mariń, J. P. Malrieu, J. L. Heully, and D. Maynau, J. Chem. Phys. 103, 2576 (1995).

${ }^{37}$ A. Scemama, E. Giner, T. Applencourt, G. David, and M. Caffarel, Quantum Package v0.6, Zenodo, 2015.

${ }^{38}$ A. Scemama and E. Giner, e-print arXiv:1311.6244 [physics.comp-ph] (2013).

${ }^{39}$ Á. Szabados, J. Chem. Phys. 134, 174113 (2011).

${ }^{40}$ S. Das, D. Mukherjee, and M. Kállay, J. Chem. Phys. 132, 074103 (2010).

${ }^{41}$ M. W. Schmidt, K. K. Baldridge, J. A. Boatz, S. T. Elbert, M. S. Gordon, J. H. Jensen, S. Koseki, N. Matsunaga, K. A. Nguyen, S. Su, T. L. Windus, M. Dupuis, and J. A. Montgomery, Jr., J. Comput. Chem. 14(11), 1347 (1993).

${ }^{42}$ P. Hiberty, J. Flament, and E. Noizet, Chem. Phys. Lett. 189, 259 (1992).

${ }^{43}$ J. P. Malrieu, N. Guihéry, C. J. Calzado, and C. Angeli, J. Comput. Chem. 28, 35 (2007).

${ }^{44}$ T. H. Dunning, J. Chem. Phys. 90, 1007 (1989).

${ }^{45}$ L. Bytautas, T. Nagata, M. S. Gordon, and K. Ruedenberg, J. Chem. Phys. 127, 164317 (2007).

${ }^{46}$ G. D. Purvis and R. J. Bartlett, J. Chem. Phys. 76, 1910 (1982).

${ }^{47}$ G. D. Purvis, R. Shepard, F. B. Brown, and R. J. Bartlett, Int. J. Quantum Chem. 23, 835 (1983).

${ }^{48}$ W. D. Laidig and R. J. Bartlett, Chem. Phys. Lett. 104, 424 (1984).

${ }^{49}$ U. S. Mahapatra, B. Datta, and D. Mukherjee, J. Chem. Phys. 110, 6171 (1999).

${ }^{50}$ M. Kállay, P. G. Szalay, and P. R. Surján, J. Chem. Phys. 117, 980 (2002).

${ }^{51}$ M. Hanrath, J. Chem. Phys. 123, 084102 (2005).

${ }^{52}$ F. A. Evangelista, W. D. Allen, and H. F. Schaefer, J. Chem. Phys. 125, 154113 (2006)

${ }^{53}$ F. A. Evangelista, E. Prochnow, J. Gauss, and H. F. Schaefer, J. Chem. Phys. 132, 074107 (2010).

${ }^{54}$ M. Hanauer and A. Köhn, J. Chem. Phys. 134, 204111 (2011).

${ }^{55} \mathrm{~J}$. Olsen, P. Jørgensen, H. Koch, A. Balkova, and R. J. Bartlett, J. Chem. Phys. 104, 8007 (1996).

${ }^{56}$ G. K.-L. Chan, M. Kállay, and J. Gauss, J. Chem. Phys. 121, 6110 (2004).

${ }^{57}$ M. Nooijen, K. R. Shamasundar, and D. Mukherjee, Mol. Phys. 103, 2277 (2005).

${ }^{58}$ J. P. Malrieu, Mol. Phys. 111, 2451 (2013).

${ }^{59}$ J. Miralles, O. Castell, R. Caballol, and J. P. Malrieu, Chem. Phys. 172, 33 (1993).

${ }^{60}$ B. Pradines, N. Suaud, and J. P. Malrieu, J. Phys. Chem. A 119, 5207 (2015). 